\title{
Encapsulation of an antifungal agent within biodegradable polymers: composition effect
}

\author{
Rima Kassab ${ }^{1}$, Dima Moussa ${ }^{1}$, Paolo Yammine ${ }^{* 1}$ \\ ${ }^{1}$ Department of Chemistry, Faculty of Sciences, University of Balamand, Tripoli, Lebanon \\ rima.kassab@balamand.edu.lb \\ dima.moussa@balamand.edu.lb \\ paolo.yammine@balamand.edu.lb \\ “Corresponding author: paolo.yammine@balamand.edu.lb
}

\section{ABSTRACT}

\begin{abstract}
Polylactic acid and poly(lactic-co-glycolic acid) are two aliphatic polyesters commonly used in drug delivery systems. Having a hydrophobic nature, they could be used for the encapsulation of hydrophobic drugs such as Amphotericin B. Drug-loaded microspheres were prepared using solvent evaporation by changing the ratio of Polylactic acid to poly(lacticco-glycolic acid) in the organic mixture. Results showed that higher drug encapsulation and drug loading values were seen for formulations having higher lactide content. This had also influenced the drug release rate which was slower at higher lactide percentage. Microspheres, which were spherical in shape and porous in structure, had homogeneous particles sizes ranging between 100 and $300 \mu \mathrm{m}$. At last, FT-IR spectra recorded in this study revealed a stable character of both polymer and drug after microencapsulation.
\end{abstract}

\section{Indexing terms/Keywords}

Polylactic acid, Poly(lactic-co-glycolic acid), Amphotericin B, drug delivery, in vitro release

\section{Academic Discipline And Sub-Disciplines}

Pharmaceutical Chemistry

\section{SUBJECT CLASSIFICATION}

Chemistry

\section{Council for Innovative Research}

Peer Review Research Publishing System

\section{Journal: Journal of Advances in Chemistry}

\author{
Vol.12, No. 3 \\ www.cirworld.com, editor@cirworld.com
}




\section{INTRODUCTION}

Much research has been focused recently on using biodegradable polymeric microspheres as novel drug delivery systems. Thermoplastic aliphatic polyesters such as Polylactic acid (PLA) and poly(lactic-co-glycolic acid) (PLGA) are most commonly used in these applications owing to their biodegradability, biocompatibility, mechanical strength, and nontoxicity[1-4] (Figure 1). Medical uses of these polymers in drug delivery involve the microencapsulation of small dug molecules as well as large biomolecules as proteins, peptides, and DNA/RNA[5-9].<smiles>CC(C)OC(=O)C(C)OC(=O)C(C)O</smiles>

(a)<smiles>COC(C)C(C)OCC(C)=O</smiles>

Fig 1: Structures of (a) PLA where $n$ is the number of units of lactic acid and (b) PLGA where $x$ and $y$ and number of units of lactic acid and glycolic acid respectively [6]

With new diseases and infections emerging worldwide, the work in the medical field is concentrating on finding suitable drug treatment methods that are highly effective and less costing. Among these, the hydrophobic small drug molecules constitute a significant class which could be extremely helpful in polymeric delivery systems. However, their use poses a great challenge in order to reach the best encapsulation and release rates. These characteristics are affected by several factors such as drug properties, its solubility in aqueous and organic media, its stability in vivo, and drug-polymer interactions [10].

Routes of drug administration mainly depend on drug properties in addition to the type of delivery system. Yet, the oral route of administration remains the most commonly used, since it is simple, painless, and dosing of the medication can be easily adjusted or terminated [10,11]. Drug delivery systems prepared to be used in oral administration must enable the protection of the drug from enzymatic degradation and remaining body organs from undesirable side effects.

Many studies have been done to encapsulate hydrophobic drugs in polymeric microspheres of PLA and PLGA. These could be prepared by different methods, among which, the emulsion solvent evaporation technique is the most widespread. However, there are several preparative variables that could affect drug encapsulation and release rates obtained; most importantly the drug solubility within the polymeric mixture [12].

Amphotericin $B(A m B)$, an antifungal hydrophobic drug, is chosen as a model drug to be encapsulated in polymeric microspheres of PLA and PLGA. This is done by changing the ratio of lactide and glycolide content in the polymeric mixture to evaluate the resulting effects on prepared microspheres.f drug content, particle size, morphology, interaction between drug and polymer, stability, drug release and cytotoxicity.

\section{EXPERIMETAL}

\subsection{Materials}

The drug Amphotericin B, the polymers Poly(DL-lactide) (MW 75,000-120,000) and poly(lactic-co-glycolic acid) (lactide:glycolide 50:50, MW 30,000-60,000), the surfactant tween 80, and Phosphate Buffered Saline (PBS) (0.2 M, pH 7.4) are purchased from Sigma-Aldrich, Chemie Germany. The solvents Dichloromethane (DCM) and Methanol (MeOH) are of analytical grade.

\subsection{Methods}

AmB-loaded PLA and PLGA microspheres were synthesized using o/w emulsion solvent evaporation method. It consisted on preparing different microspheres formulations by varying the ratios of PLA and PLGA each time in the polymeric mixture and fixing the quantity of drug used, as shows Table 1. This was done to check the effect of lactide and gycolide content, in addition to the effect of drug solubility in this polymer solution on drug encapsulation and release.

Typically, $500 \mathrm{mg}$ of polymer mixture containing PLA and PLGA at different ratios and $70 \mathrm{mg}$ drug were dissolved in an organic solution of 14:6 ml DCM:MeOH. This organic phase was added to an aqueous phase containing the surfactant Tween 80. The whole mixture was stirred for 6 hours at $1400 \mathrm{rpm}$ using a mechanical stirrer to allow solvent evaporation and microspheres formations. These were collected by filtration after washing with water and methanol, finally oven dried. 
Table 1. Ratios of PLA and PLGA in the polymeric mixture

\begin{tabular}{|l|l|}
\hline $\begin{array}{l}\text { Formulation } \\
\text { Code }\end{array}$ & $\begin{array}{l}\text { Polymer Mixture } \\
\text { PLA:PLGA (mg) }\end{array}$ \\
\hline F1 & $500: 0$ \\
\hline F2 & $400: 100$ \\
\hline F3 & $250: 250$ \\
\hline F4 & $100: 400$ \\
\hline F5 & $0: 500$ \\
\hline
\end{tabular}

Following microencapsulation, the prepared microspheres were characterized for:

Drug Encapsulation (\%DE) and Drug Loading (\%DL): Microspheres are dissolved in 7/3 ml DCM/MeOH and the drug content of each formulation is measured by UV/Vis Sectrophotometry (Microplate Spectrophotometer, Epoch Biotek, USA) at $409 \mathrm{~nm}$. The percentage drug entrapment (\%DE) and drug loading (\%DL) are calculated as follows:

$$
\begin{aligned}
& \% \mathrm{DE}=\frac{\text { Encapsulated drug mass }}{\text { Introduced drug mass }}{ }^{*} \mathbf{1 0 0} \\
& \% \mathrm{DL}=\frac{\text { Encapsulated drug mass }}{\text { Microspheres mass }}{ }^{*} \mathbf{1 0 0}
\end{aligned}
$$

Particle size: a Laser Diffraction Granulometer (LA950V2, Horiba Ltd., France) is used to determine the size of microspheres. A quantity of microspheres is suspended in water, with Tween 80 used as dispersant. The average particle size is measured in micrometers.

- Physical appearance: microspheres are examined for their physical appearance by Scanning Electron Microscopy (SEM) (LYRA3 XMU, TESCAN, Czech Republic). Microspheres are fixed to a carbon conductive tape. A coating of $10 \mathrm{~nm}$ of Platinum is applied using a sputter coater.

Fourier Transform Infrared (FT-IR) study: analysis was carried out on a FT-IR spectrometer (Frontier NIR, Perkin Elmer, USA) in order to investigate the possible chemical interactions between the drug and the polymeric matrix. Samples were mixed with IR grade $\mathrm{KBr}$ salt and pressed into pellets using a manual hydraulic press for scanning.

In vitro drug release study: The In vitro release study is carried out in PBS solution (0.2 M, pH 7.4). $25 \mathrm{mg}$ microspheres are introduced in small vial containing $25 \mathrm{ml}$ PBS, used as release medium, and maintained at $37^{\circ} \mathrm{C}$. At different time intervals, $5 \mathrm{ml}$ of the release medium is withdrawn and replaced with fresh solution. This is evaluated for its drug content at $409 \mathrm{~nm}$.

\section{RESULTS AND DISCUSSION}

Table 2 shows that higher values of DE and DL were observed for formulations with higher PLA ratios, and thus higher lactide content in the polymeric mixture. This could be explained by the fact that PLA is more hydrophobic than PLGA due to the presence of the side methyl groups, as illustrated in Figure $1[6,13,14]$. As a result, the increased size of hydrophobic region facilitates the incorporation of AmB which is hydrophobic in character [15]. In addition, the increase in hydrophobicity with increase in lactide content results in better solid-state solubility of AmB in the hydrophobic polymeric matrix $^{12}$. These reasons explain the higher DE and DL values for F1 to F3 compared to the other formulations.

Table 2. \% DE, \%DL, and average particle size of AmB microspheres formulations

\begin{tabular}{|c|c|c|c|}
\hline $\begin{array}{c}\text { Formulation } \\
\text { Code }\end{array}$ & \%DE & $\% \mathrm{DL}$ & $\begin{array}{c}\text { Size } \\
(\boldsymbol{\mu m})\end{array}$ \\
\hline F1 & 23.3 & 0.66 & 138 \\
\hline F2 & 19.7 & 0.55 & 300 \\
\hline F3 & 15.6 & 0.43 & 233 \\
\hline F4 & 16 & 0.48 & 173 \\
\hline F5 & 10.2 & 0.45 & 125 \\
\hline
\end{tabular}


Table 1 also gives that the average particle size for the prepared microspheres. It ranges between 125 and $300 \mu \mathrm{m}$. This indicates that the variation in PLA and PLGA ratios in the polymeric mixture, and thus increasing in lactide content, doesn't have any influence on particle size. It is rather the procedure used and changing the speed of mixing that leaves a direct impact on the particle size $[16,17]$. Moreover, SEM analysis presented in Figure 2 showed mcirospheres that are spherical in shape and porous in structure.

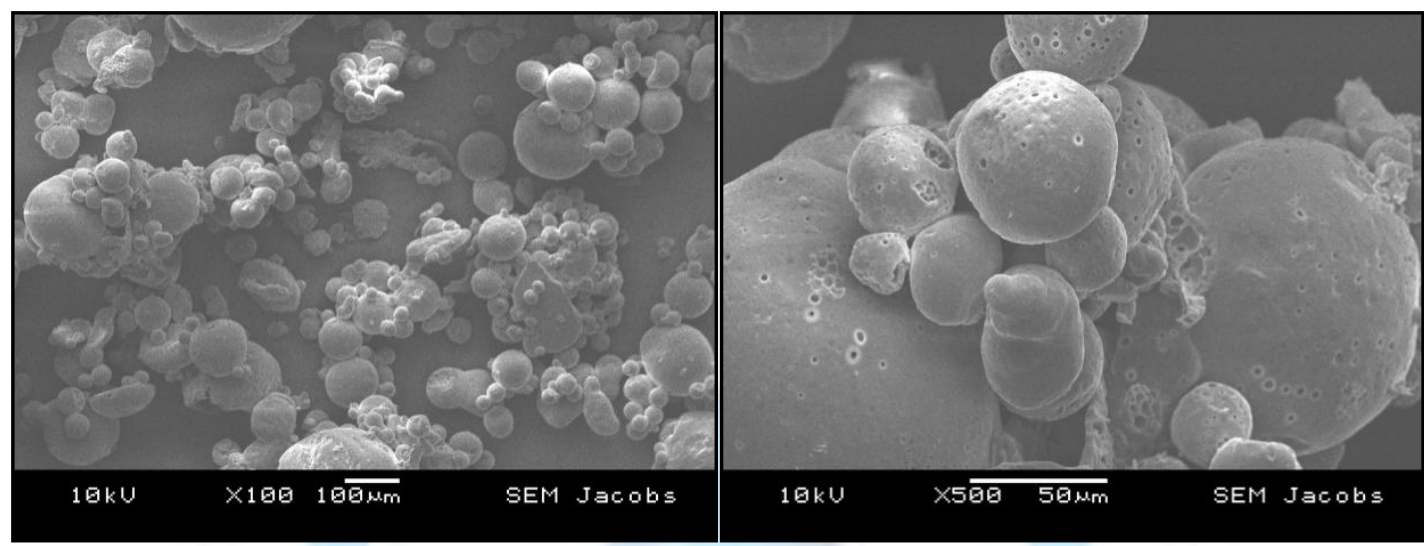

Fig 2: SEM photos of microspheres at (a) 100x and (b) 500x magnifications

Concerning in vitro drug release, Table 3 shows that the release rate is slower for formulations having the highest \%DL values. These are characterized by a higher burst release after $1 \mathrm{hr} 30 \mathrm{~min}$ in comparison to the other formulations that have a more prolonged or extended release, as shows Figure 3 . In addition, the release rate increases as \%DL decreases. At higher \%DL, higher drug quantity requires more time to be released [18,19].

\section{Table 3. Drug release time of AmB microspheres formulations}

\begin{tabular}{|l|l|l|}
\hline $\begin{array}{l}\text { Formulation } \\
\text { Code }\end{array}$ & \%DL & $\begin{array}{l}\text { Complete Release } \\
\text { Time (hrs) }\end{array}$ \\
\hline F1 & 0.66 & 30 \\
\hline F2 & 0.55 & 24 \\
\hline F3 & 0.43 & 2 hrs 30 min \\
\hline F4 & 0.48 & 7 \\
\hline F5 & 0.45 & 5 \\
\hline
\end{tabular}

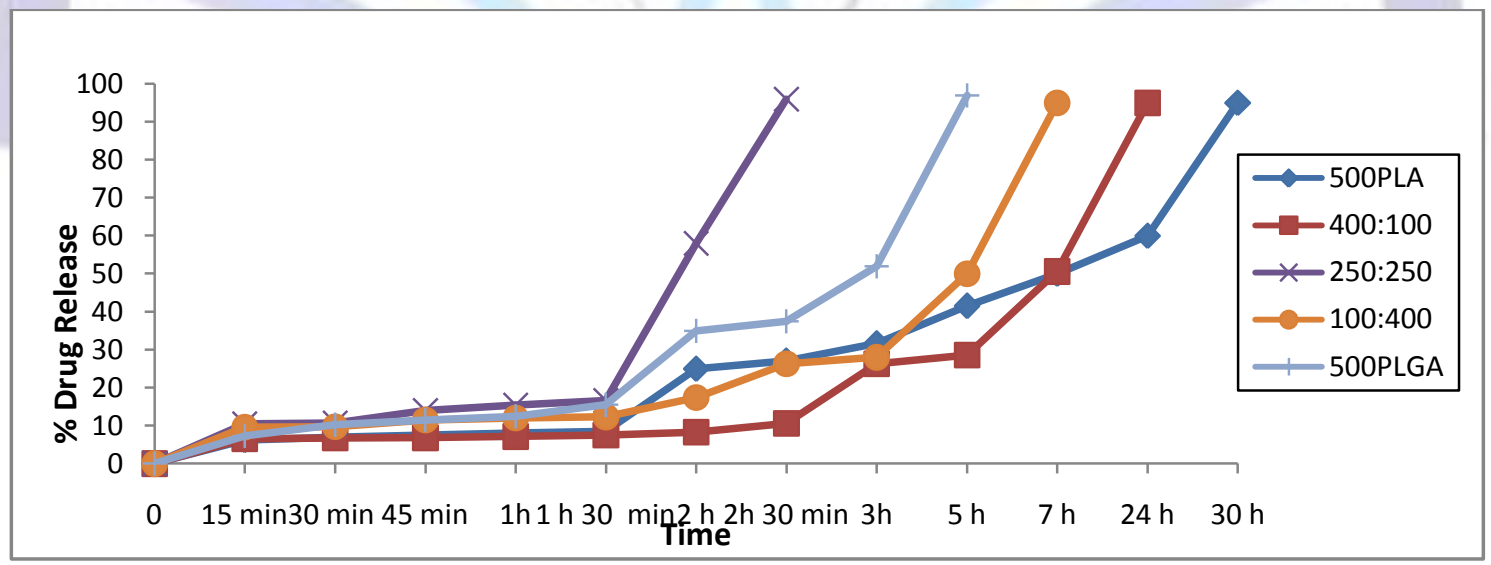

Fig 3: Drug release profile of AmB microspheres formulations

In additoon, the release rate is directly affected by the ratio of PLA and PLGA in the polymeric mixture. For formulations with higher lactide content, they have a slower release rate. This could be explained by the affinity of hydrophobic AmB to the more hydrophobic lactide-rich polymer [13,14].

Besides, polymer characteristics as the solid-state polymer-drug solubility have an influence on drug release and drug distribution between the polymeric phase and the aqueous release medium [12]. Higher polymer-drug solubility results in 
lower release rates. This is due to the higher hydrophobicity of the polymer containing more lactide, and to lower diffusion of the hydrophobic drug to the external release medium [20].

Lastly, figure 4 reveals the results of polymer-drug physico-chemical compatibility studies. FT-IR spectra were recorded for AmB, PLA/PLGA blank microspheres, and PLA/PLGA AmB-loaded microspheres. For PLA/PLGA blank microspheres, characteristic peaks are noted at $2900-3000 \mathrm{~cm}^{-1}$ for aliphatic $\mathrm{C}-\mathrm{H}$ stretching, at $1750 \mathrm{~cm}^{-1}$, for $\mathrm{C}=\mathrm{C}$ alkene stretching, at $1450 \mathrm{~cm}^{-1}$ for aliphatic C-H bending, and at $1080-1170 \mathrm{~cm}^{-1}$ for C-O ester stretching $[21,22]$. These peaks are also present in the spectrum of loaded microspheres. For $A m B$, several characteristic peaks appear at $1690 \mathrm{~cm}^{-1}$ for $C=C$ alkene, at $2940 \mathrm{~cm}^{-1}$ for $\mathrm{C}-\mathrm{H}$ methyl stretching, and the broad bank at $3300-3400 \mathrm{~cm}^{-1}$ for $-\mathrm{OH}$ alcohol and $-\mathrm{NH}_{2}$ amine groups $[23,24]$. This broad band which is absent in the spectrum of blank polymeric microspheres is present in the case of drugloaded microspheres. This indicates the presence of both drug and polymer intact in drug-loaded microspheres and the absence of any chemical interaction between the two during microencapsulation procedure.

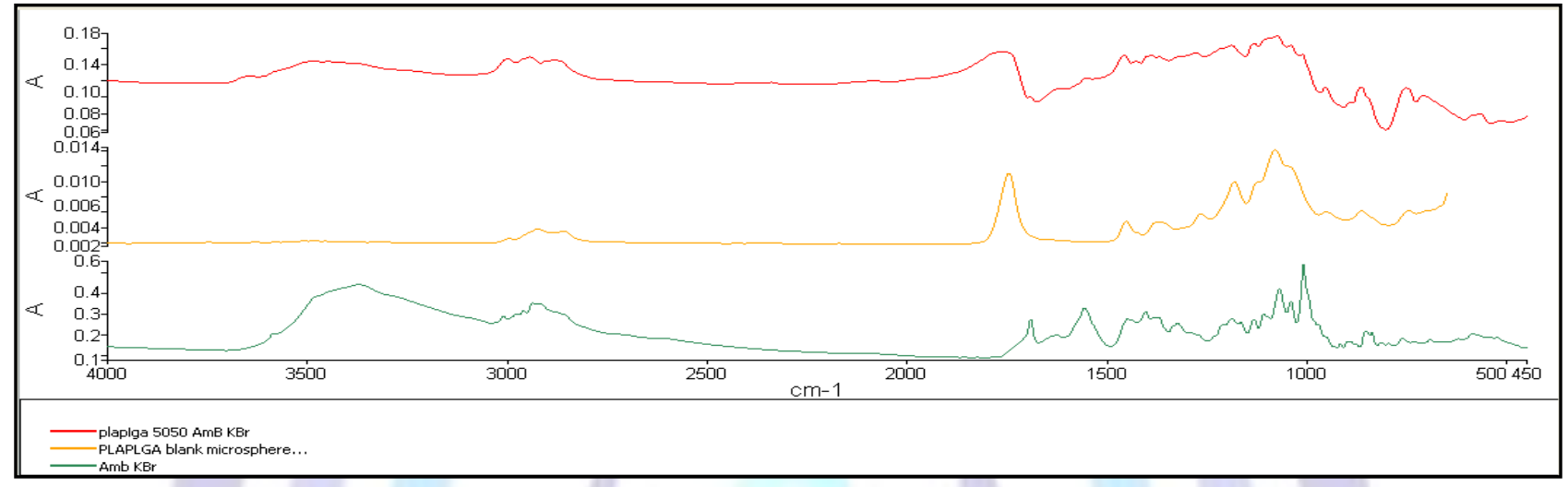

Fig 4: FT-IR spectra for AmB, PLA/PLGA blank microspheres, and PLA/PLGA AmB-loaded microspheres

\section{CONCLUSION}

The emuslion solvent evaporation technique is one of the most widespeard and successful methods for preparing drug delivery systems based on microspheres ready for oral administration. Polymeric Amphotericin B-loaded microspheres of Polylactic acid and Poly(lactid-co-glycolic acid) were formulated by varying the ratio of the two polymers in the organic mixture. This factor had a great influence on drug encapsulton and release. As the lactide or polylactic acid content increased, Amphotericin B loading and encapsulation also increased. In addition, the drug release was found to be slower with more lactide. Particle size obtained was not influenced and was more homogenous for all formulations which is expected in the case of solvent evaporation which also yields spherical and porous microspheres. Finally, FT-IR analysis revealed that both the drug and the polymeric mixture remained intact following microencapsulation.

\section{ACKNOWLEDGEMENTS}

The authors would like to express their gratitude to the Chemitsry Department at the University of Balamand for supporting this project by providing the needed chemicals and materials and and carrying out the experimental procedure.

\section{REFERENCES}

[1] Heidi, M.M., Sohn, M. J., Al-Ghananeem, A., and Delucca, P.P. 2010. Materials for pharmaceutical dosage forms: Molecular pharmaceutics and controlled release drug delivery aspects. Int. J. Mol. Sci. 11(9), 3298-3322.

[2] Sadeghi, M. 2011. Pectin-based biodegradable hydrogels with potential biomedical applications as drug delivery systems. J. Biomater. Nanobiotechnol. 2(1), 36-40.

[3] Giavaresi, G., Tschon, M., Borsari, V., Daly, J.H., Liggat, J.J., Fini, M., Bonazzi, V., Nicolini, A., Carpi, A., Morra, M., Cassinelli, C., and Giardino, R. 2004. New polymers for drug delivery systems in orthopaedics: in vivo biocompatibility evaluation. Biomed Pharmacother. 58(8), 411-7.

[4] Chandiran, I.S., Sivakumar, T., and Kumar, B.P. 2010. Preparation and evaluation of Aceclofenac loaded biodegradable microspheres. Int. J. Pharm. Biomed. Res. 1(1), 19-23.

[5] Johansen, P., Men, Y., Merkle, H.P., and Gander, B. 2000. Revisiting PLA/PLGA microspheres: an analysis of their potential in parenteral vaccination. Eur. J. Pharm. Biopharm. 50, 129-146.

[6] Jena, K.K. 2011. Biodegradable polymers (PLA and PLGA) based nanoparticles in protein and plasmid DNA delivery. Master Thesis. National Institute of Technology, Rourkela, India.

[7] Makadia, H., and Siegel, S. 2011. PLGA as biodegradable controlled drug delivery carrier. Polymers (Basel). 3(3), 1377-1397.

[8] Kumari, A., Yadav, S.K., and Yadav, S.C. 2010. Biodegradable polymeric Nanoparticles based drug delivery systems. Colloids Surf. B: Biointerfaces. 75, 1-18. 
[9] Wu, XS. 1995b. Preparation, characterizatiom and drug delivery application of microspheres based biodegradable lactic/glycoic acid polymers. In Wise D., Trantolo DJ., Altobelli DE, Yaszems MJ. (Eds). Encyclopedic handbook of biomaterials and bioengineering: Materials/applications. Marcel Dekker.

[10] Wischke,C., and Schwendeman, S. 2008. Principles of encapsulating hydrophobic drugs in PLA/PLGA microparticles. Int. J. Pharm. 364, 298-327.

[11] Takami, T., and Murakami, Y. 2011. Development of PEG-PLA/PLGA microparticles for pulmonary drug delivery prepared by a novel emulsification technique assisted with amphiphilic block copolymers. Colloids Surf. B: Biointerfaces. 87, 433-438.

[12] Panyam, J., Williams, D., Dash, A., Leslie-Pelecky, D., and Labhasetwar, V. 2004. Solid-State Solubility Influences Encapsulation and Release of Hydrophobic Drugs from PLGA/PLA Nanoparticles. J. Pharm. Sci. 93(7), 1804-1814.

[13] Chakravarti, S. 2008. Effect of size of PLGA and chitosan-PLGA particles on the cellular association, cytotoxicity, and anti-tumor efficacy of Paclitaxel.PhD dissertation. University of Nebraska.

[14] Jain, R. 2000. The manufacturing techniques of various drug loaded biodegradable PLGA devices. Biomaterials. 21, 2475-2490.

[15] Zhou, W., Wang, Y., Jian, J., and Song, S. 2013. Self-aggregated nanoparticles based on amphiphilic poly(lactic acid)grafted-chitosan copolymer for ocular delivery of Amphotericin B. Int. J. Nanomed. 8, 3715-3728.

[16] Mukherjee, B., Santra, K., Pattnaik, G., and Ghosh, S.2008. Preparation, characterization and in-vitro evaluation of sustained release protein-loaded nanoparticles based on biodegradable polymers. Int. J. Nanomed. 3(4), 487-496.

[17] Moussa, D., Kassab, R., and Yammine, P. 2014. Study of different processing parameters for Polylactic acid microspheres formulations. Int. J. Pharm. Sci. Res. 5(10), 4176-4181.

[18] Yuce, M., and Canefe, K. 2008. Indomethacin-loaded microspheres: Preparation, characterization and in-vitro evaluation regarding ethylcellulose matrix material. Turkish J. Pharm. Sci. 5, 129-142.

[19] Jyothi,V., Prasanna,M., Sakarkan,N., Prabha,S., Ramaiah,S., and Srawan,Y. 2010. Microencapsulation techniques, factors influencing encapsulation efficiency. J. Microencapsul. 27(3), 187-197.

[20] Minghetti, P., Cilurzo, F., Casiraghi, A., and Montanari, L. 1999. Application of viscometry solubility parameters in miconazole patches development. Int. J. Pharm. 190(1), 91-101.

[21] Erbetta, C., Alves, R., Resende, J., Freitas, R., and De Souza, R. 2012. Synthesis and characterization of PLGA copolymer. J. Biomater. Nanobiotechnol. 3, 208-225.

[22] Kiremitci-Gumusderelioglu, M., and Deniz, G. 1999. Synthesis, Characterization and in Vitro Degradation of Poly(DLLactide)/Poly(DL-Lactide-co-Glycolide). Turk. J. Chem. 23, 153-161.

[23] Gagos, M., and Arczewska, M. 2010. Spectroscopic studies of molecular organization of antibiotic Amphotericin B in Monolayers and dipalmitoylphosphatidylcholine lipid multibilayers. Biochim. Biophys. Acta- General Subjects. 1798(11), 2124-2130.

[24] Das, S., and Suresh, P. 2011. Nanosuspension: a new vehicle for the improvement of the delivery of drugs to the ocular surface. Nanomed. Nanotechnol. Biol. Med. 7(2), 242-247. 